\title{
Resposta Espacial de uma Antena Inteligente Linear sob os Efeitos do Acoplamento Mútuo
}

\author{
Manoel J. L. Alves, Marcelo S. Alencar e Marcelo P. Sousa
}

\begin{abstract}
Resumo-Os arranjos de antenas fornecem uma diretividade elevada e controlável, útil para sistemas de comunicações. Projetos desse tipo não são triviais, devido à característica não-linear dos arranjos nos diversos campos de aplicações, requerendo o uso de técnicas de otimização, como os algoritmos supervisionados LMS (least mean square) e RLS (recursive least square). Com o propósito de otimizar o controle eletrônico da resposta espacial de arranjos de antenas por algoritmos supervisionados foi desenvolvida uma ferramenta computacional capaz de posicionar o lóbulo principal e rejeitar interferências com direções de chegada conhecida, considerando os efeitos do acoplamento mútuo entre os elementos do arranjo.
\end{abstract}

Palavras-Chave-Arranjo de antenas, Acoplamento mútuo, Antenas adaptativas, Resposta espacial.

Abstract - The antenna arrays provide a high and controllable directivity, useful for communication systems. Those designs are not trivial, due to the non-linear characteristic of the arrays in the several fields of applications, requiring the use of optimization techniques, like the supervised algorithms LMS (least mean square) and RLS (recursive least square). With the purpose of optimizing the electronic control of the space response of antenna arrays by supervised algorithms a computational tool was developed, and is capable of to position the main lobe and to reject interferences with known direction-of-arrival, considering the effects of mutual coupling among the elements of the array.

Keywords-Antenna array, mutual coupling, adaptive antennas, space response.

\section{INTRODUÇÃO}

As antenas inteligentes são sistemas de antenas que reagem às mudanças do ambiente dinamicamente, com o objetivo de fornecer um sinal de maior qualidade e gerar um melhor aproveitamento das faixas de freqüência nas comunicações sem fio. Esses sistemas envolvem processamento de sinais induzidos sobre um arranjo de sensores tais como: antenas, microfones e hidrofones. Eles têm aplicações nas áreas de radar, sonar, imagiologia médica e comunicações [1]. As antenas inteligentes têm a propriedade de filtragem espacial, que as permite receber energia a partir de uma direção particular, enquanto minimiza a recepção em outra.

O processamento de sinais, a partir de diferentes antenas, envolve a amplificação de cada sinal antes de sua combinação. $\mathrm{O}$ ganho de cada amplificador estabelece as propriedades do arranjo de antenas. Para obter o melhor cancelamento de interferências possível, os ganhos desses amplificadores devem ser ajustados. O objetivo é maximizar a SNR do sinal na saída. Para um arranjo com uma determinada resposta na direção do

Manoel J. L. Alves, IESAM - Instituto de Estudos Superiores da Amazônia, Belém, PA. Marcelo S. Alencar e Marcelo P. Sousa, Iecom - Instituto de Estudos Avançados em Comunicações, Universidade Federal de Campina Grande, Campina Grande, PB. E-mails: \{jacinto, malencar, marporsou $\} @$ iecom.org.br. Este trabalho foi financiado pelo IESAM, Iecom e CNPq. sinal desejado, isto é alcançado minimizando a potência média de saída do processador sujeito às limitações especificadas [1].

O comportamento de estruturas de arranjo de antenas é normalmente modelado combinando os diagramas de radiação dos elementos de antena individuais por meio de superposição vetorial (em amplitude e fase). No entanto, quando pares de antenas estão próximos uns dos outros, se uma ou ambas as antenas estão no modo de transmissão e/ou recepção, parte da energia que se destina a uma antena é acoplada às outras e é referida como acoplamento mútuo (AM). A quantidade de AM depende das características de irradiação de cada antena, da separação relativa entre o par de antenas e da orientação relativa de cada antena. Na maioria dos métodos utilizando modelos de superposição, os efeitos do AM entre os elementos do arranjo são ignorados, reduzindo o desempenho do sistema. No entanto, resultados do desempenho de um arranjo adaptativo mostram que, aplicando as correntes complexas de excitação obtidas na convergência de um algoritmo adaptativo em condições ideais (sem acoplamento), o desempenho resultante do arranjo adaptativo em uma situação real, em que os efeitos de AM estão envolvidos, pode ser muito pior [2].

No processamento de arranjos adaptativos, medidas de compensação são adotadas contra a influência do AM. No entanto, o desempenho de um arranjo adaptativo é mais realista comparado com aquele na ausência do AM. Baseado [3] em uma rede de transformação equivalente, o desempenho de arranjos adaptativos com compensação do AM foi analisado. $\mathrm{O}$ arranjo adaptativo pode minimizar a interferência, mas a potência de saída do ruído muda com a direção do sinal desejado, o que provoca a degradação da razão sinal-ruído mais interferência (SINR - signal-to-interference plus noise ratio). Portanto, é principalmente o ruído térmico, após a compensação do AM, que degrada a SINR.

$\mathrm{O}$ arranjo de antenas adaptativo é uma ferramenta para sistemas de comunicações sem fio, uma vez que pode aumentar significativamente a capacidade e a banda do canal [4]. Vários algoritmos de conformação de feixes e métodos de estimação da direção de chegada (DOA - direction of arrival) foram desenvolvidos [4]-[6]. Também, foi mostrado que o AM distorce as repostas espaciais de arranjos conformadores e afeta algumas importantes métricas dos arranjos adaptativos [7]. Por isso, o AM deve ser contabilizado para que sistemas de antenas adaptativos práticos possam alcançar um desempenho semelhante aos sistemas ideais. Várias modelagens de AM e métodos para compensar o AM têm sido propostos para minimizar os efeitos do AM. Algumas dessas aproximações implementam a compensação do AM nas amostras de forma que o sistema possa obter amostras como no caso ideal, e então obter as mesmas soluções do caso prático [7]-[10]. 
Este artigo encontra-se dividido da seguinte forma: a Seção II apresenta a modelagem dos efeitos do acoplamento mútuo de um arranjo de antenas. A Seção III mostra o processo de adaptação dos pesos para um arranjo adaptativo (antena inteligente). Os resultados de simulações das respostas espaciais com e sem os efeitos do AM para um arranjo adaptativo são apresentados na Seção IV. A Seção V apresenta as conclusões do artigo.

\section{Modelagem dos Efeitos do Acoplamento Mútuo de um ARRANJo de ANTEnAS}

Um arranjo de antenas linear genérico de $M$ elementos idênticos é ilustrado na Fig. 1. Os elementos são espaçados de uma distância $d$, um em relação ao outro, e são terminados em cargas casadas.

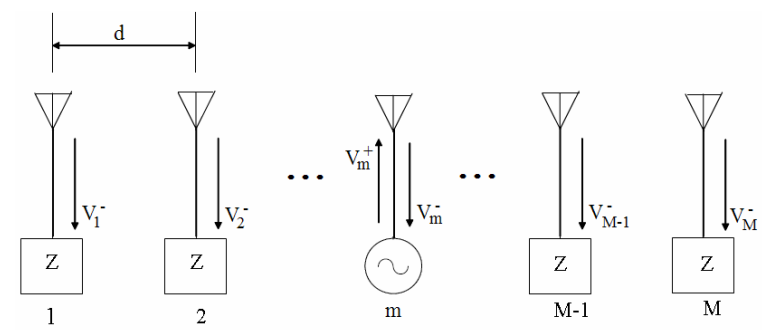

Fig. 1. Esquema elétrico de um arranjo de antenas linear com o elemento $m$ excitado por uma fonte de tensão.

Para obter a resposta espacial de um elemento de antena, somente um dos $M$ elementos é excitado. Essa situação é ilustrada na Fig. 1 para o elemento $m$, em que $m=1,2, \ldots, M$. $\mathrm{O}$ efeito do acoplamento mútuo é modelado pela presença de ondas de tensão que se propagam a partir dos elementos não excitados para dentro das cargas.

A amplitude da onda de tensão é relacionada aos campos elétrico e magnético irradiados $E_{e}$ e $H_{e}$, respectivamente, de um elemento de antena isolado, por meio de

$$
\begin{aligned}
& E_{e}(\theta)=V_{e} S_{e}(\theta) \frac{e^{-j k_{\circ}} r}{r}, \\
& H_{e}(\theta)=\frac{V_{e}}{\eta} S_{e}(\theta) \frac{e^{-j k_{\circ}} r}{r},
\end{aligned}
$$

em que $V_{e}$ é a amplitude da onda de tensão da excitação, $k_{\circ}=2 \pi / \lambda$ e $S_{e}(\theta)$ é o fator de elemento, ou seja, é a resposta espacial de um único irradiador. A impedância característica do vácuo, $\eta_{\mathrm{o}}$, é dada por

$$
\eta_{\circ}=\sqrt{\frac{\mu_{\circ}}{\epsilon_{\circ}}}
$$

em que $\mu_{\circ}$ é a permeabilidade magnética do vácuo e $\epsilon_{\circ}$ é a permissividade elétrica do vácuo.

Assume-se que o elemento irradia um único modo e que um único modo propaga-se na rede de excitação. O elemento $M$ do arranjo linear pode ser visto como o acesso $M$, caracterizado por uma matriz de espalhamento $M \times M$, em que os coeficientes de espalhamento relacionam as amplitudes da onda de tensão dos elementos de acordo com

$$
S_{m \ell}=\left.\frac{V_{m}^{-}}{V_{\ell}^{+}}\right|_{V_{k=0, k \neq \ell}^{+}} \quad \text { para } m, \ell, k=1,2, \ldots, M .
$$

Essa equação mostra que o coeficiente de espalhamento $S_{m \ell}$ é obtido comparando a amplitude da onda de tensão no elemento $m$, a partir do vácuo, com a amplitude da onda de tensão que excita o elemento $\ell$. Para essa comparação, nenhum dos elementos, com a exceção do elemento $\ell$, está sendo excitado. Todos esses elementos são terminados em cargas casadas. Esse processo é modelado na Fig. 2.

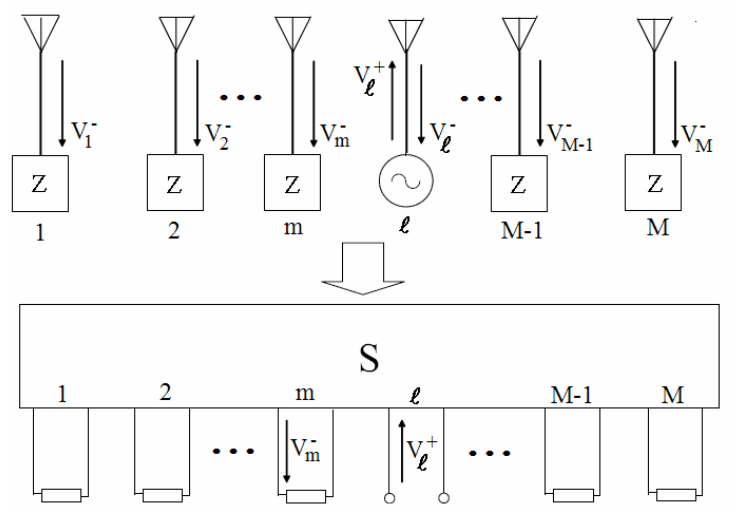

Fig. 2. Representação do equivalente elétrico de um arranjo de antenas linear com $M$ elementos.

Considere $V_{m}^{+}$e $V_{m}^{-}$as amplitudes das ondas incidente e refletida no elemento $m$, respectivamente. Com o uso de (4), é determinada a amplitude da onda de tensão refletida no elemento $m$

$$
V_{m}^{-}=\sum_{\ell=1}^{M} S_{m \ell} V_{\ell}^{+}
$$

A tensão total sobre a antena de elemento $\ell, V_{\ell}$, é a soma das amplitudes da onda de tensão incidente e refletida

$$
V_{\ell}=V_{\ell}^{+}+V_{\ell}^{-} \text {. }
$$

A corrente total é dada por

$$
I_{\ell}=I_{\ell}^{+}+I_{\ell}^{-}=\frac{1}{Z_{\circ}}\left(V_{\ell}^{+}-V_{\ell}^{-}\right),
$$

em que $Z_{\circ}$ é a impedância característica da linha de transmissão.

Supondo que a impedância característica da linha de transmissão conectada ao elemento de antena, $Z_{\circ}$, seja igual a um, i.e., uma impedância característica normalizada. Então, $I_{\ell}=V_{\ell}^{+}-V_{\ell}^{-}$.

Para que o feixe da antena do arranjo linear de $M$ elementos seja direcionado a $\theta=\theta_{\circ}$, é aplicada uma diferença de fase, satisfazendo

$$
V_{\ell}^{+}=e^{-j k_{\circ}(\ell-1) d \operatorname{sen}\left(\theta_{\circ}\right)},
$$

em que a referência de fase está no elemento 1.

$\mathrm{O}$ coeficiente de reflexão de varredura (coeficiente de reflexão em cada elemento do arranjo como uma função do ângulo de varredura $\left.\theta_{\circ}\right), \Gamma_{m}\left(\theta_{\circ}\right)$, do elemento $m$, em sua 
posição no arranjo, com todos os elementos excitados e corretamente em fase, é determinado com o auxílio de (5) e (8)

$\Gamma_{m}\left(\theta_{\circ}\right)=\frac{V_{m}^{-}}{V_{m}^{+}}=e^{j k_{\circ}(m-1) d \operatorname{sen}\left(\theta_{\circ}\right)} \sum_{\ell=1}^{M} S_{m \ell} e^{-j k_{\circ}(\ell-1) d \operatorname{sen}\left(\theta_{\circ}\right)}$.

O campo elétrico irradiado do arranjo de antena linear de $M$ elementos, com todos os elementos excitados, $E_{a}\left(\theta_{\circ}\right)$, resulta em

$$
E_{a}(\theta)=E_{e}(\theta) \sum_{m=1}^{M} V_{m} e^{j k_{\circ}(m-1) d \operatorname{sen}(\theta)} .
$$

Esse campo é obtido pela regra da multiplicação de diagramas, em que se multiplica o fator de elemento com o fator de arranjo (resposta espacial de um arranjo de $M$ irradiadores isotrópicos). No entanto, os efeitos de acoplamento mútuo não são levados em conta.

Para induzir os efeitos do acoplamento mútuo, se relaciona a amplitude da onda de tensão $V_{m}$, ao coeficiente de reflexão de varredura, $\Gamma(\theta)$, usando (6) e (9). Assim,

$$
V_{m}=V_{m}^{+}+V_{m}^{-}=\left[1+\Gamma_{m}\left(\theta_{\circ}\right)\right] V_{m}^{+} .
$$

Substituindo este resultado junto (8) em (10), chega-se ao campo elétrico irradiado do arranjo de antena completamente excitado, dado por

$$
E_{a}(\theta)=E_{e}(\theta) \sum_{m=1}^{M}\left[1+\Gamma_{m}\left(\theta_{\circ}\right)\right] e^{j k_{\circ}(m-1) d\left[\operatorname{sen}(\theta)-\operatorname{sen}\left(\theta_{\circ}\right)\right]},
$$

e, em fase para $\theta=\theta_{\circ}$, resulta em

$$
E_{a}\left(\theta_{\circ}\right)=E_{e}\left(\theta_{\circ}\right) \sum_{m=1}^{M}\left[1+\Gamma_{m}\left(\theta_{\circ}\right)\right] \text {. }
$$

A substituição de (1) em (13), resulta em

$$
E_{a}\left(\theta_{\circ}\right)=V_{e} S_{e}\left(\theta_{\circ}\right) \sum_{m=1}^{M}\left[1+\Gamma_{m}\left(\theta_{\circ}\right)\right] \frac{e^{-j k_{\circ} r}}{r} .
$$

De maneira similar, acha-se o campo magnético irradiado do arranjo de antena completamente excitado

$$
H_{a}\left(\theta_{\circ}\right)=\frac{V_{e}}{\eta} S_{e}\left(\theta_{\circ}\right) \sum_{m=1}^{M}\left[1-\Gamma_{m}\left(\theta_{\circ}\right)\right] \frac{e^{-j k_{\circ} r}}{r} .
$$

A função ganho $G(\theta)$ é dada por

$$
G(\theta)=\frac{P(\theta)}{P_{\text {tot }} / 4 \pi}
$$

em que $P(\theta)$ é a potência irradiada na direção $\theta$ e $P_{t o t}$ é a potência incidente total disponível.

A densidade de potência média temporal $\bar{W}$ dos campos irradiados pode ser calculada como

$$
\bar{W}(r)=\frac{1}{2} \Re e\left\{\bar{E}_{a}(r) \times \bar{H}_{a}^{*}(r)\right\}=\frac{1}{2} \Re e\left\{E_{a} H_{a}^{*}\right\} \bar{u}_{r},
$$

em que $\overline{\mathbf{u}}_{r}$ é um vetor unitário na direção $r$.

A potência irradiada $P(\theta)$, por unidade de ângulo sólido é determinada por

$$
P(r)=P(\theta)=\left|r^{2} \bar{W}(r)\right| .
$$

A potência irradiada na direção $\theta_{\circ}$ torna-se

$$
\begin{aligned}
P\left(\theta_{\circ}\right) & =V_{e}^{2} S_{e}^{2}(\theta) \frac{1}{2 \eta}\left\{\sum_{m=1}^{M}\left[1+\Gamma_{\mathrm{m}}\left(\theta_{\circ}\right)\right]\right\} \\
& \times\left\{\sum_{m=1}^{M}\left[1-\Gamma_{\mathrm{m}}^{*}\left(\theta_{\circ}\right)\right]\right\} .
\end{aligned}
$$

Uma vez que foi assumida uma impedância característica normalizada, a potência total disponível é

$$
P_{t o t}=M \frac{V_{e}^{2}}{2}
$$

de modo que o ganho do arranjo completamente excitado na direção $\theta_{\circ}$ é determinado por

$$
G_{a}\left(\theta_{\circ}\right)=\frac{4 \pi S_{e}^{2}\left(\theta_{\circ}\right)}{M \eta}\left(M^{2}-\left|\sum_{m=1}^{M} \Gamma_{m}\left(\theta_{\circ}\right)\right|^{2}\right) .
$$

Assim, baseado no coeficiente de reflexão de varredura $\Gamma_{m}$, que pode ser obtido a partir de medições de acoplamento mútuo por pares, entre os elementos do arranjo e o diagrama de radiação do elemento isolado, pode-se obter o ganho do arranjo de antenas $G_{a}\left(\theta_{\circ}\right)$, na direção do feixe de varredura.

\section{Processo de AdAPTAÇÃo dos Pesos do ARranjo ADAPTATIVO}

Os arranjos de antenas em que a resposta espacial é formada de acordo com certo critério ótimo, são chamados de antenas inteligentes. As antenas inteligentes têm sido alternativamente chamadas de arranjos conformadores digitais de feixes ou arranjos adaptativos.

As respostas espaciais das antenas adaptativas são controladas por algoritmos baseados em certos critérios. Neste trabalho, o critério usado é o da minimização do erro quadrático médio (MSE - mean-square error), orientando para um sinal de interesse, cancelando os sinais interferentes, ou localizando um emisssor em movimento. A implementação desses algoritmos pode ser feita eletronicamente por meio de dispositivos analógicos, mas é geralmente mais fácil usar processamento digital de sinais, resultando em uma conformação digital de feixes.

Quando os algoritmos usados são adaptativos, esse processo é chamado conformação adaptativa de feixe. A principal vantagem do confomador digital de feixe é que a diferença de fase e a ponderação do arranjo podem ser efetuadas nos dados digitalizados em vez de ser implementado em hardware.

\section{A. Mínimo Erro Quadrático Médio}

Um meio alternativo para otimizar os pesos do arranjo adaptativo é obtido pela minimização do erro quadrático médio (MSE - mean-square error). A Fig. 3 ilustra uma maneira de minimizar o erro durante a iteração dos pesos do arranjo.

O sinal $d(k)$ é o sinal de referência. Preferivelmente, o sinal de referência é idêntico ao sinal desejado $s(k)$ ou é altamente correlacionado com $s(k)$ e descorrelacionado com os sinais interferentes $i(k)$. Se $s(k)$ não é claramente diferente 


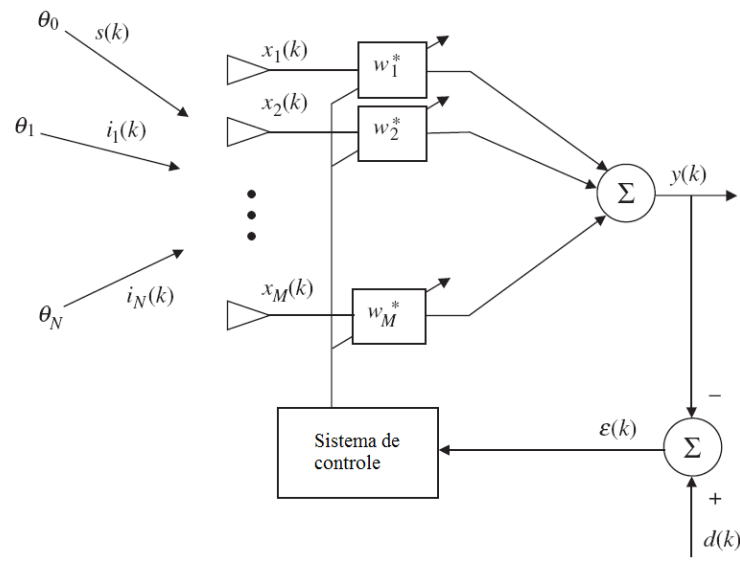

Fig. 3. Sistema adaptativo com minimização do erro quadrático médio.

dos sinais interferentes, a técnica do mínimo MSE não opera corretamente. O sinal $\varepsilon(k)$ é o sinal erro dado por

$$
\varepsilon(k)=d(k)-\overline{\mathbf{w}}^{H} \overline{\mathbf{x}}(k)
$$

e o MSE é dado por

$$
\begin{aligned}
& |\varepsilon(k)|^{2}=\varepsilon(k) \varepsilon^{*}(k)=\left(d(k)-\overline{\mathbf{w}}^{H} \overline{\mathbf{x}}(k)\right)\left(d^{*}(k)-\overline{\mathbf{w}} \overline{\mathbf{x}}^{*}\right), \\
& |\varepsilon(k)|^{2}=|d(k)|^{2}-2 d(k) \overline{\mathbf{w}}^{H} \overline{\mathbf{x}}(k)+\overline{\mathbf{w}}^{H} \overline{\mathbf{x}}(k) \overline{\mathbf{x}}^{H}(k) \overline{\mathbf{w}} .
\end{aligned}
$$

Nesse artigo é omitida a notação de dependência temporal $k$. Assim, tomando o valor esperado de ambos os lados e simplificando a expressão, obtém-se

$$
E\left[|\varepsilon|^{2}\right]=E\left[|d|^{2}\right]-2 \overline{\mathbf{w}}^{H} \overline{\mathbf{r}}+\overline{\mathbf{w}}^{H} \overline{\mathbf{R}}_{x x} \overline{\mathbf{w}},
$$

em que as seguintes correlações são definidas:

$$
\begin{gathered}
\overline{\mathbf{r}}=E\left[d^{*} \cdot \overline{\mathbf{x}}\right]=E\left[d^{*} \cdot\left(\overline{\mathbf{x}}_{s}+\overline{\mathbf{x}}_{i}+\overline{\mathbf{n}}\right)\right], \\
\overline{\mathbf{R}}_{x x}=E\left[\overline{\mathbf{x}} \overline{\mathbf{x}}^{H}\right]=\overline{\mathbf{R}}_{s s}+\overline{\mathbf{R}}_{u u}, \\
\overline{\mathbf{R}}_{s s}=E\left[\overline{\mathbf{x}}_{s} \overline{\mathbf{x}}_{s}^{H}\right], \\
\overline{\mathbf{R}}_{u u}=\overline{\mathbf{R}}_{i i}+\overline{\mathbf{R}}_{n n} .
\end{gathered}
$$

A Equação (25) é uma função quadrática do vetor peso $\overline{\mathbf{w}}$. Essa função é chamada superfície de desempenho ou função custo e é uma superfície quadrática no espaço $M$-dimensional. No entanto, uma vez que os pesos ótimos proporcionam o mínimo MSE, o extremo é o mínimo dessa função.

\section{B. Mínimo quadrado médio (LMS)}

O algoritmo LMS é um algoritmo que se baseia no gradiente [11]. Os algoritmos baseados no gradiente assumem uma superfície de desempenho quadrática. Quando a superfície de desempenho é uma função quadrática dos pesos do arranjo, a superfície de desempenho (função custo) $J(\bar{w})$ tem a forma de um hiperparabolóide tendo um mínimo. Uma das maneiras para estabelecer o mínimo é pelo uso do método do gradiente (Método de Lagrange). Dessa forma, pode-se estabelecer a superfície de desempenho encontrando o MSE. O erro, como indicado na Fig. 3, é

$$
\varepsilon(k)=d(k)-\overline{\mathbf{w}}^{H}(k) \overline{\mathbf{x}}(k) .
$$

O erro ao quadrado é dado como

$$
|\varepsilon(k)|^{2}=\left|d(k)-\overline{\mathbf{w}}^{H}(k) \overline{\mathbf{x}}(k)\right|^{2} .
$$

Como calculado em (31), a função custo resulta em

$$
J(\overline{\mathbf{w}})=D-2 \overline{\mathbf{w}}^{H} \overline{\mathbf{r}}+\overline{\mathbf{w}}^{H} \overline{\mathbf{R}}_{x x} \overline{\mathbf{w}},
$$

em que $D=E\left[|d|^{2}\right]$. Dessa forma, pode ser empregado o método do gradiente para localizar o mínimo em (32). Logo, tem-se

$$
\nabla_{\overline{\mathbf{w}}}(J(\overline{\mathbf{w}}))=2 \overline{\mathbf{R}}_{x x} \overline{\mathbf{w}}-2 \overline{\mathbf{r}} .
$$

O mínimo ocorre quando o gradiente é nulo. Desse modo, a solução para os pesos é a solução ótima de Wiener dada por

$$
\overline{\mathbf{w}}_{o t}=\overline{\mathbf{R}}_{x x}^{-1} \overline{\mathbf{r}} .
$$

A solução em (34) é baseada no conhecimento de todas as estatísticas do sinal e, portanto, no cálculo da matriz de autocorrelação.

Em geral, não se sabe as estatísticas do sinal e, portanto, deve-se recorrer ao cálculo da matriz de autocorrelação $\left(\overline{\mathbf{R}}_{x x}\right)$ do arranjo e ao vetor de correlação cruzada $(\overline{\mathbf{r}})$ do sinal sobre um intervalo de amostras ou para cada instante de tempo. As estimativas instantâneas desses valores são determinadas por

$$
\hat{\mathbf{R}}_{x x}(k) \approx \overline{\mathbf{x}}(k) \overline{\mathbf{x}}^{H}(k),
$$

$\mathrm{e}$

$$
\hat{\mathbf{r}} \approx d^{*}(k) \overline{\mathbf{x}}(k) .
$$

Entretanto, pode ser empregado uma técnica iterativa chamada o método da descida mais íngrime (steepest descent) para aproximar o gradiente da função custo. A direção da descida mais íngrime é na direção oposta ao vetor gradiente. $\mathrm{O}$ método da descida mais íngrime pode ser aproximado em termos dos pesos usando o método LMS [12], [13]. A aproximação iterativa do método steepest descent é dada por

$$
\overline{\mathbf{w}}(k+1)=\overline{\mathbf{w}}(k)-\frac{1}{2} \mu \nabla_{\overline{\mathbf{w}}}(J(\overline{\mathbf{w}}(k))),
$$

em que $\mu$ é o parâmetro de passo e $\nabla_{\overline{\mathbf{w}}}$ é o gradiente da superfície de desempenho.

O gradiente da superfície de desempenho é obtido a partir de (33). Portanto, substituindo as aproximações de autocorrelação e correlação cruzada instantâneas, tem-se a solução LMS

$$
\begin{gathered}
\overline{\mathbf{w}}(k+1)=\overline{\mathbf{w}}(k)-\mu\left[\hat{\mathbf{R}}_{x x} \overline{\mathbf{w}}-\hat{\mathbf{r}}\right], \\
\overline{\mathbf{w}}(k+1)=\overline{\mathbf{w}}(k)+\mu e^{*}(k) \overline{\mathbf{x}}(k),
\end{gathered}
$$

em que $e(k)=d(k)-\overline{\mathbf{w}}^{H}(k) \overline{\mathbf{x}}(k)$ é o sinal de erro.

\section{Mínimos quadrados recursivo (RLS)}

No algoritmo RLS, a matriz de autocorrelação $\left(\hat{\mathbf{R}}_{x x}\right)$ e o vetor de correlação cruzada $(\overline{\mathbf{r}})$ requeridos podem ser calculados recursivamente. Desse modo, pode-se reescrever a matriz de autocorrelação e o vetor de correlação cruzada como:

$$
\hat{\mathbf{R}}_{x x}(k)=\sum_{i=1}^{k} \overline{\mathbf{x}}(i) \overline{\mathbf{x}}^{H}(i),
$$




$$
\overline{\mathbf{r}}=\sum_{i=1}^{k} d^{*}(i) \overline{\mathbf{x}}(i)
$$

em que $k$ é a última amostra temporal e $\hat{\mathbf{R}}_{x x}(k), \overline{\mathbf{r}}(k)$ são as correlações estimadas que terminam na amostra temporal $k$.

Ambos os somatórios (39) e (40) utilizam janelas retangulares, assim, eles consideram igualmente todas as amostras temporais anteriores. No entanto, se as fontes de sinais podem mudar ou mover lentamente com o passar do tempo, pode ser preciso desenfatizar as primeiras amostras de dados e enfatizar as mais recentes. Isso é conseguido por meio da modificação de (39) e (40), tal que se esqueça as primeiras amostras temporais. Isso é chamado uma estimativa ponderada. Assim,

$$
\begin{gathered}
\hat{\mathbf{R}}_{x x}(k)=\sum_{i=1}^{k} \alpha^{k-i} \overline{\mathbf{x}}(i) \overline{\mathbf{x}}^{H}(i), \\
\hat{\mathbf{r}}(k)=\sum_{i=1}^{k} \alpha^{k-i} d^{*}(i) \overline{\mathbf{x}}(i),
\end{gathered}
$$

em que $\alpha$ é o fator de esquecimento.

$\mathrm{O}$ fator de esquecimento é também às vezes referido como o fator de ponderação exponencial [14], com $\alpha$ uma constante positiva tal que $0 \leq \alpha \leq 1$. Quando $\alpha=1$, retoma-se o algoritmo mínimos quadrados ordinário. Também, $\alpha=1$ indica memória infinita. Separando o somatório de (41) e (42) em dois termos: o somatório para valores até $i=k-1$ e o último termo para $i=k$, resulta em

$$
\begin{aligned}
\hat{\mathbf{R}}_{x x}(k) & =\alpha \hat{\mathbf{R}}_{x x}(k-1)+\overline{\mathbf{x}}(k) \overline{\mathbf{x}}^{H}(k), \\
\hat{\mathbf{r}}(k) & =\alpha \hat{r}(k-1)+d^{*}(k) \overline{\mathbf{x}}(k) .
\end{aligned}
$$

Deste modo, os futuros valores para a estimativa da matriz de correlação e estimativa do vetor de correlação podem ser encontrados usando valores anteriores.

Portanto, pode-se calcular recursivamente as mais recentes estimativas de correlação, usando (43) para obter uma relação recursiva para a inversa da matriz de correlação. O primeiro passo segue a dedução em [14]. Então, recorrendo ao teorema de Sherman-Morrison-Woodbury (SMW) [15] determina-se a inversa de (43). Assim, repetindo o teorema de SMW, obtémse

$$
\hat{\mathbf{R}}_{x x}^{-1}(k)=\alpha^{-1} \hat{\mathbf{R}}_{x x}^{-1}(k-1)-\alpha^{-1} \overline{\mathbf{g}}(k) \overline{\mathbf{x}}^{H}(k) \hat{\mathbf{R}}_{x x}^{-1}(k-1) .
$$

A Equação (45) é conhecida como a Equação de Riccati para o método dos mínimos quadrados recursivo.

A solução ótima de Wiener é repetida para cada iteração $k$, podendo-se substitui-la em (44)

$$
\begin{gathered}
\overline{\mathbf{w}}(k)=\hat{\mathbf{R}}_{x x}^{-1}(k) \hat{\mathbf{r}}(k), \\
\overline{\mathbf{w}}(k)=\alpha \hat{\mathbf{R}}_{x x}^{-1}(k) \hat{\mathbf{r}}(k-1)+\hat{\mathbf{R}}_{x x}^{-1}(k) \overline{\mathbf{x}}(k) d^{*}(k) .
\end{gathered}
$$

Substituindo (45) na primeira matriz de autocorrelação inversa de (46), obtém-se

$\overline{\mathbf{w}}(k)=\overline{\mathbf{w}}(k-1)-\overline{\mathbf{g}}(k) \overline{\mathbf{x}}^{H}(k) \overline{\mathbf{w}}(k-1)+\hat{\mathbf{R}}_{x x}^{-1}(k) \overline{\mathbf{x}}(k) d^{*}(k)$.

Finalmente, resultando em

$$
\overline{\mathbf{w}}(k)=\overline{\mathbf{w}}(k-1)+\overline{\mathbf{g}}(k)\left[d^{*}(k)-\overline{\mathbf{x}}^{H}(k) \overline{\mathbf{w}}(k-1)\right] .
$$

\section{Resultados de Simulações}

As respostas espaciais no plano-H (plano azimutal $(\phi)$ ) para arranjos de antenas adaptativos lineares com diferentes dimensões, espaçamentos e direções considerando os efeitos do AM entre os elementos do arranjo, foram investigadas. As simulações com os algoritmos LMS e RLS foram realizadas com o sinal desejado na direção $\phi=125^{\circ}$ e o sinal interferente na direção $\phi=30^{\circ}$. Além disso, ocorreu atualização dos pesos nos algoritmos, enquanto o mínimo erro quadrático médio $[\varepsilon(k)]$ era maior do que o critério de parada de $1 \times 10^{9}$. Também, nessas simulações, o número de iterações usado foi de 5050 amostras e o tempo de processamento com os algoritmos LMS e RLS, respectivamente, de 0,0881 e 0,2915 segundos.

As Figs. 4 e 5 representam as respostas espaciais com e sem AM para arranjos adaptativos de cinco e dez elementos, respectivamente, e espaçamentos entre os elementos do arranjo iguais a $0,25 \lambda$ e $0,50 \lambda$ e SNR $=15 \mathrm{~dB}$. Nesse caso o processamento adaptativo de sinais foi realizado com o uso do algoritmo LMS. Nas Figs. 4 e 5 é observado que há a formação de feixe do lóbulo principal para a resposta espacial do arranjo para o espaçamento igual a $0,50 \lambda$, no caso sem AM. No entanto, quando é considerado o acoplamento de um elemento adjacente sobre o outro, caso com AM, a resposta espacial do arranjo é modificada. Ou seja, não há formação do feixe principal.

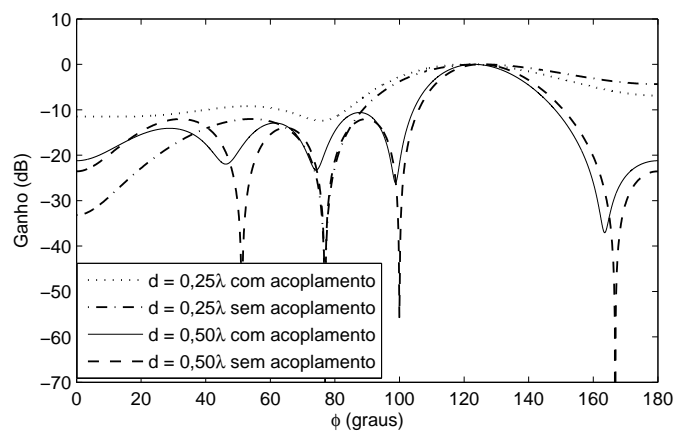

Fig. 4. Resposta espacial no plano-H de um arranjo de antenas adaptativo com cinco elementos, utilizando o algoritmo LMS.

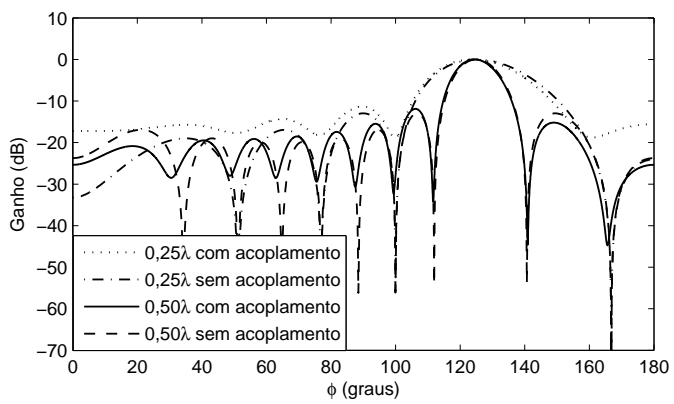

Fig. 5. Resposta espacial no plano-H de um arranjo de antenas adaptativo com dez elementos, utilizando o algoritmo LMS.

As Figs. 6 e 7 representam as respostas espaciais com e sem acoplamento mútuo para arranjos adaptativos de cinco 
e dez elementos, respectivamente, e espaçamentos entre os elementos do arranjo iguais a $0,25 \lambda$ e $0,50 \lambda$ e $\mathrm{SNR}=15$ dB. Nesse caso o processamento adaptativo foi realizado com o uso do algoritmo RLS. Nas Figs. 6 e 7 é mostrado que há a formação de feixe do lóbulo principal para a resposta espacial do arranjo para o caso sem AM (espaçamento igual a $0,50 \lambda$ ), enquanto que, para o caso com AM, em que é considerado o efeito do acoplamento de um elemento adjacente sobre o outro modifica-se a resposta espacial do arranjo. Assim, fica claro para ambos os arranjos (cinco e dez elementos) que o arranjo adaptativo com AM tem uma resposta espacial mais realista [5], [16], [17], pois é diferente do que é apresentado pela resposta espacial, quando o AM é desconsiderado.

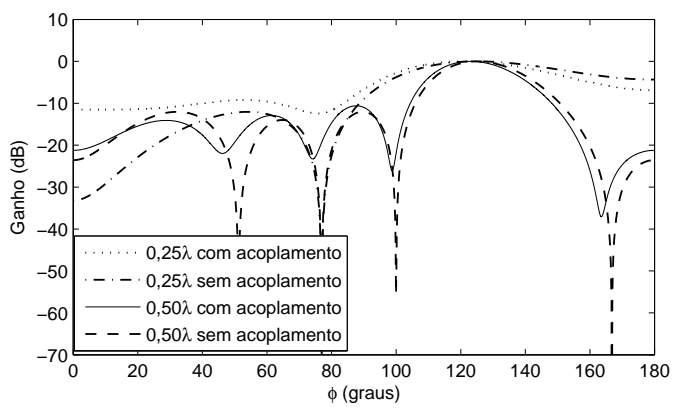

Fig. 6. Resposta espacial no plano-H de um arranjo de antenas adaptativo com cinco elementos, utilizando o algoritmo RLS.

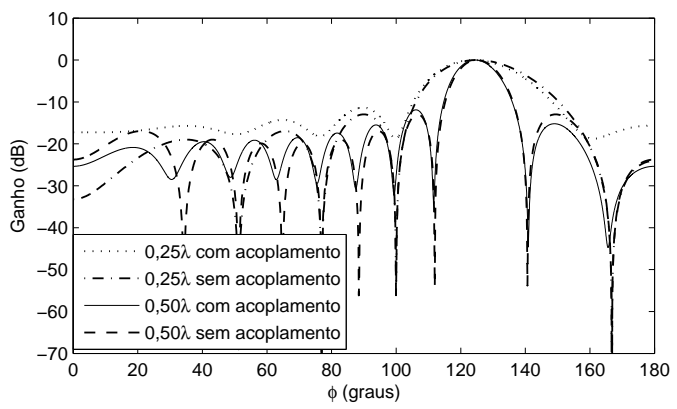

Fig. 7. Resposta espacial no plano-H de um arranjo de antenas adaptativo com dez elementos, utilizando o algoritmo RLS.

\section{CONCLUSÕES}

A caracterização do arranjo de antenas foi realizada supondo que no arranjo o elemento de antena está a uma distância $d$ um em relação ao outro e os efeitos do AM entre os elementos do arranjo é da mesma ordem. Dessa forma, baseado no coeficiente de reflexão obtido a partir de medições do AM por pares de elementos do arranjo, e a resposta espacial de irradiação do elemento isolado, o ganho $(\mathrm{dB})$ do arranjo na direção do sinal desejado foi determinado.

Também foi mostrada a teoria de um arranjo de antenas adaptativo linear que consiste de um arranjo de antenas e uma unidade de processamento digital de sinais (algoritmos LMS ou RLS) que otimiza dinamicamente a resposta espacial do arranjo adaptativo de acordo com a mudança do ambiente eletromagnético. A principal vantagem do arranjo adaptativo é que a mudança de fase e a ponderação do arranjo podem ser realizadas com os dados digitalizados em vez de serem implementadas em hardware.

Os resultados das simulações considerando os efeitos do AM em arranjos de antenas dipolos lineares (comprimento da antena dipolo $-L=\lambda / 2$ ), para diferentes espaçamentos e dimensões, foram obtidos por meio do processamento adaptativo de sinais. Os algoritmos supervisionados LMS e RLS foram usados na conformação de feixe, a fim de obter a resposta espacial (ganho em dB) do sinal na direção desejada, plano$H$, de acordo com certo critério ótimo.

\section{REFERÊNCIAS}

[1] L. C. Godara, Smart Antennas, CRC Press LCC, New York, 2004.

[2] P. Ioannides, C. A. Balanis, "Mutual Coupling in Adaptive Circular Arrays", Antennas and Propagation Society International Symposium, 2004. IEEE, Vol. 1, Issue, 20-25 June 2004, Page(s) 403-406 Vol. 1.

[3] L. R. Feng et al., "Analysis of the Performance of Adaptive Arrays with Mutual Coupling Compensation", Antennas and Propagation Society International Symposium, 2006. IEEE, pp. 4781-4784, Octuber 2006.

[4] G. V. Tsoulos, "Smart antennas for mobile communication systems: Benefits and challenges", Electron. Commun. Eng. J., pp. 84-94, Apr. 1999.

[5] I. J. Gupta, A.A. Ksienski, "Effect of Mutual Coupling on the Performance of Adaptive Arrays", IEEE Transactions on Antennas and Propagation, v.31, p.785-791, September 1983.

[6] H. Steyskal, J. S. Herd, "Mutual Coupling Compensation in Small Array Antennas", IEEE Transactions on Antennas and Propagation, vol. 38, pp.1971-1975, December 1990

[7] R. S. Adve, T. K. Sarkar, "Compensation for the Effects of Mutual Coupling on Direct Data Domain Adaptive Algoritms", IEEE Transactions on Antennas and Propagation, 48(1):86-95, January 2000.

[8] K. R. Dandekar, H. Ling, G. Xu, "Experimental Study of Mutual Coupling Compensation in Smart Antenna Applications", IEEE Transactions Wireless Communications, vol.1, pp. 480-487, July 2002.

[9] H. T. Hui, "Improved Compensation for the Mutual Coupling Effect in a Dipole Array for Direction Finding", IEEE Transactions on Antennas and Propagation, vol. 51, pp. 2498-2503, September 2003.

[10] Z. Huang, C. A. Balanis, C. R. Birtcher, "Mutual Coupling in Beamforming with Uniform Circular Array: Theory and Experiment", IEEE Transactions on Antennas and Propagation, vol. 54, pp. 3082-3086, November 2006.

[11] R. A. Monzingo, T. W. Miller, Introduction to Adaptive Arrays, Wiley, New York, 1980.

[12] B. Widrow, M. Hoff, "Adaptive Switch Circuits", IRE Wescom, Convention Record, Part 4, pp. 96-104, 1960.

[13] B. Widrow et al., "Adaptive Antenna Systems", Proceedings of the IEEE, Vol. 55, December, 1967.

[14] S. Haykin, Adaptive Filter Theory, 4th Ed., Prentice Hall, New York, 2002.

[15] G. H. Golub, C. H. Van Loan, Matrix Computations, The Johns Hopkins University Press, 3d. Ed., 1996.

[16] Z. Huang, C. A. Balanis, "The MMSE Algorithm and Mutual Coupling for Adaptive Arrays", IEEE Transactions on Antennas and Propagation, 56(5):1292-1296, May 2008

[17] M. J. L. Alves, M. S. Alencar, "Effects of Mutual Coupling in Smart Antenna Arrays", In: IEEE International Microwave and Optics Conference - IMOC2007, 2007, Salvador, Brasil. Proceedings of the IEEE International Microwave and Optics Conference. Piscataway, USA:IEEE/MTTS, 2007.v1.p.1-5 